\title{
Simultaneous Multi-Slice fMRI using spiral trajectories
}

Citation for published version (APA):

Zahneisen, B., Poser, B. A., Ernst, T., \& Stenger, A. V. (2014). Simultaneous Multi-Slice fMRI using spiral trajectories. Neuroimage, 92, 8-18. https://doi.org/10.1016/j.neuroimage.2014.01.056

Document status and date:

Published: 15/05/2014

DOI:

10.1016/j.neuroimage.2014.01.056

Document Version:

Publisher's PDF, also known as Version of record

Document license:

Taverne

Please check the document version of this publication:

- A submitted manuscript is the version of the article upon submission and before peer-review. There can be important differences between the submitted version and the official published version of record.

People interested in the research are advised to contact the author for the final version of the publication, or visit the DOI to the publisher's website.

- The final author version and the galley proof are versions of the publication after peer review.

- The final published version features the final layout of the paper including the volume, issue and page numbers.

Link to publication

\footnotetext{
General rights rights.

- You may freely distribute the URL identifying the publication in the public portal. please follow below link for the End User Agreement:

www.umlib.nl/taverne-license

Take down policy

If you believe that this document breaches copyright please contact us at:

repository@maastrichtuniversity.nl

providing details and we will investigate your claim.
}

Copyright and moral rights for the publications made accessible in the public portal are retained by the authors and/or other copyright owners and it is a condition of accessing publications that users recognise and abide by the legal requirements associated with these

- Users may download and print one copy of any publication from the public portal for the purpose of private study or research.

- You may not further distribute the material or use it for any profit-making activity or commercial gain

If the publication is distributed under the terms of Article $25 \mathrm{fa}$ of the Dutch Copyright Act, indicated by the "Taverne" license above, 


\title{
Simultaneous Multi-Slice fMRI using spiral trajectories
}

\author{
Benjamin Zahneisen ${ }^{\mathrm{a}, *}$, Benedikt A. Poser ${ }^{\mathrm{b}}$, Thomas Ernst ${ }^{\mathrm{a}}$, Andrew V. Stenger ${ }^{\mathrm{a}}$ \\ a University of Hawaii, Department of Medicine, John A. Burns School of Medicine, Honolulu, HI, USA \\ ${ }^{\mathrm{b}}$ Maastricht University, Maastricht, Netherlands
}

\section{A R T I C L E I N F O}

Article history:

Accepted 31 January 2014

Available online 8 February 2014

\begin{abstract}
A B S T R A C T
Parallel imaging methods using multi-coil receiver arrays have been shown to be effective for increasing MRI acquisition speed. However parallel imaging methods for fMRI with 2D sequences show only limited improvements in temporal resolution because of the long echo times needed for BOLD contrast. Recently, Simultaneous Multi-Slice (SMS) imaging techniques have been shown to increase fMRI temporal resolution by factors of four and higher. In SMS fMRI multiple slices can be acquired simultaneously using Echo Planar Imaging (EPI) and the overlapping slices are un-aliased using a parallel imaging reconstruction with multiple receivers. The slice separation can be further improved using the "blipped-CAIPI" EPI sequence that provides a more efficient sampling of the SMS 3D k-space. In this paper a blipped-spiral SMS sequence for ultra-fast fMRI is presented. The blipped-spiral sequence combines the sampling efficiency of spiral trajectories with the SMS encoding concept used in blipped-CAIPI EPI. We show that blipped spiral acquisition can achieve almost whole brain coverage at $3 \mathrm{~mm}$ isotropic resolution in $168 \mathrm{~ms}$. It is also demonstrated that the high temporal resolution allows for dynamic BOLD lag time measurement using visual/motor and retinotopic mapping paradigms. The local BOLD lag time within the visual cortex following the retinotopic mapping stimulation of expanding flickering rings is directly measured and easily translated into an eccentricity map of the cortex.
\end{abstract}

(c) 2014 Elsevier Inc. All rights reserved.

\section{Introduction}

Sub-second whole brain imaging is of particular interest in Blood Oxygen Level Dependent (BOLD) functional Magnetic Resonance Imaging (fMRI) (Feinberg et al., 2010; Posse et al., 2012; Zahneisen et al., 2012). Faster acquisitions with higher temporal sampling of the BOLD time course provide several advantages. One immediate benefit is the increased sensitivity in detecting functional activation for a given stimulation duration simply by acquiring a greater number of samples. Using more sophisticated data analyses, improved sampling also allows for direct observation of the underlying BOLD hemodynamic response function (HRF). If the full HRF is recorded, regional variations in shape, onset, lag time or other general characteristics can be incorporated into the model fitted to the observed data leading to lower residuals and increased statistical significance (Dilharreguy et al., 2003). Furthermore, cardiac and respiration components of the physiological noise signal can also be measured directly and filtered out, possibly improving the overall temporal SNR. Lastly, higher volume acquisition rates freeze out head motion for improved image quality.

The most commonly used strategy to accelerate image acquisition times involves parallel imaging methods (Griswold et al., 2002; Pruessmann et al., 1999; Sodickson and Manning, 1997), where nonacquired k-space data are estimated using receiver array coil sensitivity

\footnotetext{
* Corresponding author at: University of Hawaii, JABSOM, The Queen's Medical Center,

1356 Lusitana Street, 7th floor, Honolulu, HI 96813, USA.

E-mail address: benjamin.zahneisen@gmail.com (B. Zahneisen).
}

information. Current commercially available receiver coil designs (i.e. "soccer ball" patterns with 32 channels (Wiggins et al., 2006)) provide sensitivity variation along all three spatial dimensions. This favors the use of three-dimensional (3D) sequences that can be accelerated along the in-plane and slice-select spatial dimensions. Standard Cartesian two-dimensional (2D) acquisitions however can only be accelerated along the phase encoding direction and thus make use of coil sensitivity information along a single dimension. Although two- to four-fold inplane acceleration is easily feasible with $2 \mathrm{D}$ methods, the savings in sampling time especially for 2D single-shot BOLD fMRI or diffusionweighted EPI are in practice very marginal due to the need for an adequate echo time (TE) to generate BOLD contrast. If the accelerated acquisition time is decreased beyond the optimal TE, this results in reduced BOLD amplitude, which decreases the ability to distinguish between noise and functional activation. Fast 3D single shot sequences based on Cartesian (Posse et al., 2012; Witzel, 2008) and non-Cartesian trajectories (Assländer et al., 2013; Zahneisen et al., 2012) volume acquisition times below $100 \mathrm{~ms}$ have been demonstrated. The drawback of single shot 3D methods is that the available time for k-space data acquisition is limited to approximately two times the $\mathrm{T} 2{ }^{*}$-decay time constant. Therefore these sequences can only provide moderate spatial resolution (3-4 mm $)^{3}$ and are prone to off-resonance artifacts.

Recently, Simultaneous Multi-Slice (SMS) imaging was introduced for EPI based fMRI (Feinberg et al., 2010; Nunes et al., 2006; Setsompop et al., 2012) bridging the gap between 2D multi-slice and 3D single shot imaging. In SMS EPI multiple thin, spatially separated slices are excited at once using a multi-band RF excitation pulse 
(Larkman et al., 2001; Moeller et al., 2010). The length of an SMS readout is the same as those for standard 2D acquisitions, and so the temporal acceleration factor is equal to the number of simultaneously excited slices. Recent work on SMS acquisitions proposed the so-called "blipped CAIPI" EPI method (Setsompop et al., 2012), which is the EPI analog of the CAIPIRINHA (Breuer et al., 2006) technique. The "blipped-CAIPI" EPI method use z-gradient blips played out simultaneously with the in-plane y-gradient (phase encoding) blips in order to provide zencoding along the slice direction and for more efficient use of the coil sensitivities. This allows for improved reconstruction of simultaneously excited slices and lower g-noise penalty (Setsompop et al., 2012). In essence, blipped-CAIPI exploits in-plane and slice direction coil sensitivity variation to reliably separate more slices than the coil sensitivities along slice direction alone allow. Slice acceleration factors of eight and even up to 16 have been shown to be possible on standard 32 channel coils (Chen et al., 2013) when no concurrent in-plane undersampling is used. An overview of the recent developments in ultra-fast SMS imaging can be found in Feinberg and Setsompop (2013).

Here, we present a blipped-spiral trajectory design for fast nonCartesian SMS imaging where z-gradient blips are applied for each revolution of the spiral in analogy to blipped-CAIPI EPI. Both the blipped versions of spiral and EPI trajectories share the concept of distributing $\mathrm{k}$-space encoding for SMS imaging along all three dimensions. The SMS encoding process is furthermore presented using a 3D k-space picture that describes optimal Cartesian and non-Cartesian sampling (Zahneisen et al., in press; Zhu et al., 2013) in a generalized way. The blipped-spiral trajectory combines the advantageous properties of spiral fMRI with the advantages of single-shot SMS acquisitions. For a comprehensive overview of spiral fMRI please see the review by G. Glover (2012). We show that a blipped spiral acquisition can achieve almost whole brain coverage at $3 \mathrm{~mm}$ isotropic resolution in $168 \mathrm{~ms}$. We also show that blipped-spirals have a more benign response to static offresonances compared to a stacked-spiral (Assländer et al., 2013; Irarrazabal and Nishimura, 1995) approach that was also applied to non-Cartesian SMS imaging (Riemenschneider et al., 2013). Finally the sequence is demonstrated using visual/motor and retinotopic mapping paradigms for high temporal resolution fMRI at $3 \mathrm{~T}$.

\section{Theory}

\section{Simultaneous Multi-Slice imaging and 3D k-space}

We demonstrated previously that a 3D encoding space formalism can be used to describe SMS encoding and the subsequent reconstruction of $N$ simultaneously excited slices (Zahneisen et al., in press). In contrast to a 2D SMS description where the encoding problem is explained as individual slices that fold onto each other during the readout, the slice direction now corresponds to a second phase encoding direction of a 3D encoding space ( $\mathrm{k}$-space), with a field of view along the slice select direction given by the number of excited slices times their separation. By defining a Cartesian 3D k-space that corresponds to full sampling of the simultaneously excited slices, non-Cartesian sampling strategies (either fully sampled or accelerated) can be applied readily to SMS imaging. We first present a brief summary of the SMS 3D kspace framework for SMS imaging. See Zahneisen et al. (in press) for a more complete description.

Given $N$ simultaneously excited slices with slice separation $\Delta z$, the kspace in the slice direction $z$ is defined by

$\mathrm{FOV}_{z}=N \Delta z \quad$ and $\quad \Delta k_{z}=\frac{1}{\mathrm{FOV}_{z}} \quad$ and $\quad k_{z}^{\max }=\Delta k_{z} \frac{N}{2}$.

$\mathrm{FOV}_{z}$ gives the field of view along the slice direction. The slice direction k-space component $k_{z}$ together with $k_{x}$ and $k_{y}$, for the field of view FOV $V_{x y}$ and resolution $N_{x y}$ in the $x$ - and $y$-directions, span the 3D SMS $\mathrm{k}$-space for full sampling of the in-plane and slice directions. In this work, SMS 3D k-space refers to a k-space that describes SMS imaging and is defined by Eq. (1). If the Cartesian SMS 3D k-space is fully sampled, a straightforward inverse 3D Fourier transform will reconstruct all of the simultaneous slices. If the SMS 3D k-space is undersampled, as in standard SMS imaging, coil sensitivity information and parallel imaging reconstruction techniques are needed for un-aliased reconstruction. The ratio of the fully sampled to accelerated SMS 3D k-space is the total acceleration factor $R_{\text {tot }}$ which can be distributed between different k-space directions. Note that the SMS 3D k-space framework can be used to better understand all previously published Cartesian methods using SMS excitation. For example, using an unmodified 2D readout as in Moeller et al. (2010) corresponds to sampling only the central plane in the SMS 3D k-space and therefore provides only limited slice acceleration. By adding z-blips during the 2D readout as in Setsompop et al. (2012), the SMS 3D k-space sampling density is more uniformly distributed in 3D and thus allows for greater SMS acceleration.

Fig. 1 shows an example of $N=5$ simultaneously excited slices with a slice separation $\Delta z=20 \mathrm{~mm}$ and the corresponding SMS 3D k-space with $N$ Cartesian $k_{x}-k_{y}$-planes at locations in generalized units depicted as black circles. As an example of an accelerated non-Cartesian acquisition a stacked-spiral trajectory is plotted in Fig. 1b. The stacked-spiral trajectory is typically generated using a series of 2D spirals separated by z-gradient blips with k-space increment $\Delta k_{z}^{s}$. The number of 2D spirals in the stack is given as $N_{z}$. In this example $\Delta k_{z}^{S}=\frac{5}{3} \Delta k_{z}$ and the reduction factor along $k_{z}$ is $R_{z}=\frac{\Delta k_{z}^{s}}{\Delta k_{z}}=5 / 3$. The reduction factor along $k_{z}$ can also be expressed in terms of the number of SMS slices and the number of spirals in the stack and is then given as $R_{z}=N / N_{z}$. It describes how undersampled the $k_{z}$ direction is. Sufficient coil sensitivity variation is needed to compensate for this. For example if $R_{z}=1$ then no coil sensitivity information along $\mathrm{z}$ is needed which corresponds to a k-space sampling pattern where the number of slices and the number of sampled k-space planes (stacks) are identical. Note that because of the relative rotations of each spiral along $k_{z}$ any in-plane aliasing will be shifted from slice to slice analogous to CAIPIRINHA methods. The inplane aliasing can be removed using parallel imaging reconstruction with in-plane sensitivity. In-plane acceleration is also possible with the stacked-spiral trajectory by decreasing $\mathrm{FOV}_{x y}$ by an in-plane reduction factor equal to $R_{x y}$. In-plane acceleration effectively reduces the number of spiral rotations per plane.

\section{Blipped-spiral trajectories}

Like the stacked-spiral trajectory, the blipped-spiral method is based on a planar spiral in the $k_{x}-k_{y}$-plane, orthogonal to $k_{z}$ (Fig. 2a). Unlike the stacked-spiral trajectory, however, the gradient blips in the $z$-direction are added after every full rotation of the $x$ - and $y$-gradients (Figs. 2b and c). This difference is critical to the off-resonance response of the trajectory, as we will show later. The blip moment still determines the actual $\mathrm{k}$-space increment $\Delta k_{z}^{s}$ and we assume that the trajectory starts at k-space location $-k_{z}^{\max }$ after the application of a pre-winder blip prior to the spiral. Similar to blipped CAIPI implementations in EPI, 2-4 blips are typically applied depending on the degree of sampling required along $k_{z}$. After $k_{z}^{\max }$ is reached a larger gradient pulse is substituted for the blip which rephases the k-space coordinate back to $-k_{z}^{\max }$. The initial 2D spiral is then distributed over $N_{z}=N / R_{z} \mathrm{k}-$ space planes in the $k_{z}$ direction. Like the stacked-spiral trajectory, the reduction factor along $k_{z}$ is given as $R_{z}=\Delta k_{z}^{S} / \Delta k_{z}$ where $\Delta k_{z}$ is given by Eq. (1). In standard spiral imaging full sampling of the $k_{x}-k_{y}$ plane with resolution $N_{x y}$ requires $N_{x y} / 2$ revolutions. Consequently, full sampling of $N$ simultaneous slices requires that the generating spiral be $N$-fold oversampled resulting in $N N_{x y} / 2$ revolutions in order to ensure that sampling of each $k_{z}$-plane fulfills the Nyquist criterion, as depicted by the gray line in Fig. 2d. The effective in-plane acceleration factor depends on the oversampling factor of the initial spiral and the number of $\mathrm{k}$-space planes in the $k_{z}$ direction. For any given SMS parameters $N$ and 


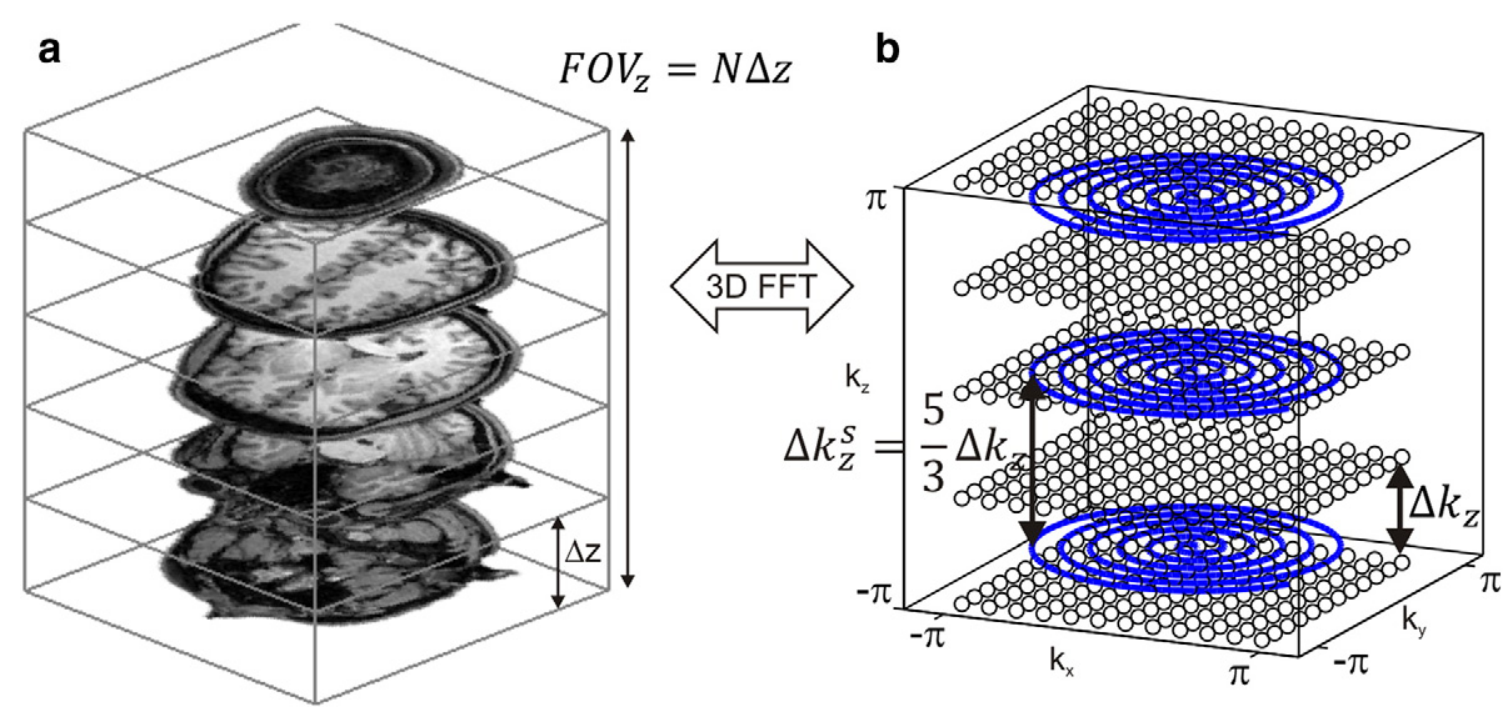

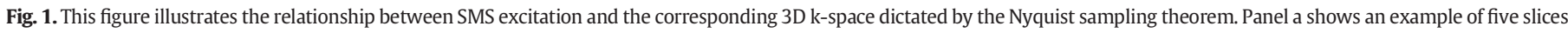

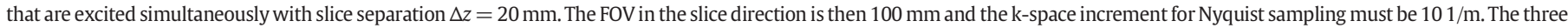
spirals (blue lines in panel b) are an example for accelerated non-Cartesian sampling of the full k-space since only three out of the five k-space planes are sampled.

$\Delta z$, the k-space increment in the slice direction is identical for the blipped versions of spiral and EPI SMS trajectories.

Fig. 3 shows an example for $N=5$ slices and in-plane resolution $N_{x y}=48$ in generalized k-space units. In Fig. 3a full sampling is shown where each of the five $k_{z}$-planes contains a spiral with 24 revolutions. Figs. 3b-d depict different scenarios of in-plane acceleration versus slice acceleration, with a total acceleration factor of five in all cases. In Fig. $3 \mathrm{~b}$ only a single $k_{z}$-plane is sampled with 24 revolutions.
This trajectory is fully sampled in the $x y$-direction and fivefold undersampled in the slice direction $\left(R_{z}=5\right)$. Restoration of missing $\mathrm{k}$-space points entirely relies on receiver coil sensitivity variation in the slice direction. In contrast, the trajectory in Fig. 3c is fully sampled in the slice direction and fivefold undersampled in the in-plane direction. No coil sensitivity variation in the slice direction is needed to restore missing k-space samples and therefore $R_{z}=1$. A more practical distribution is shown in Fig. 3d with threefold in-plane $R_{z}=5 / 3$ in
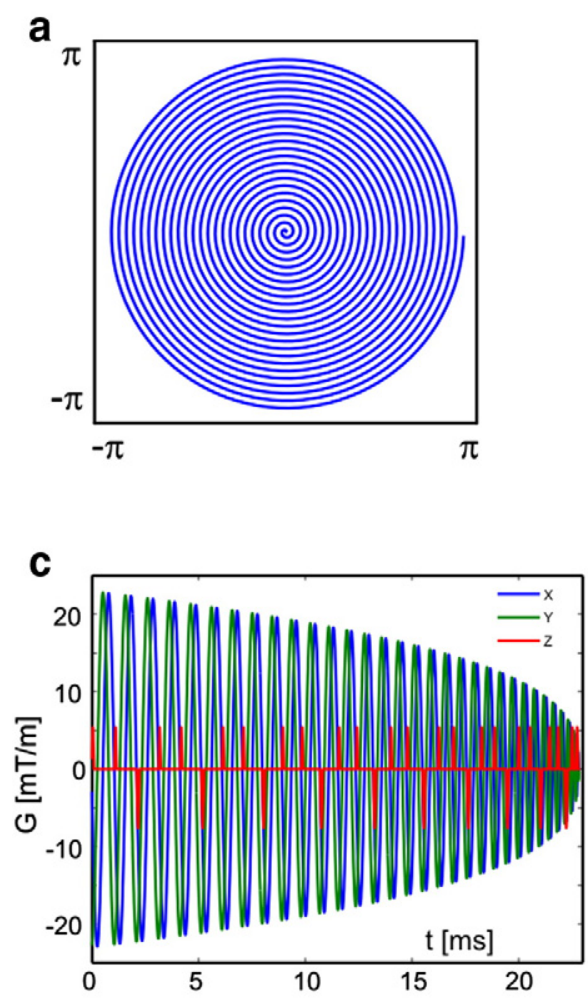
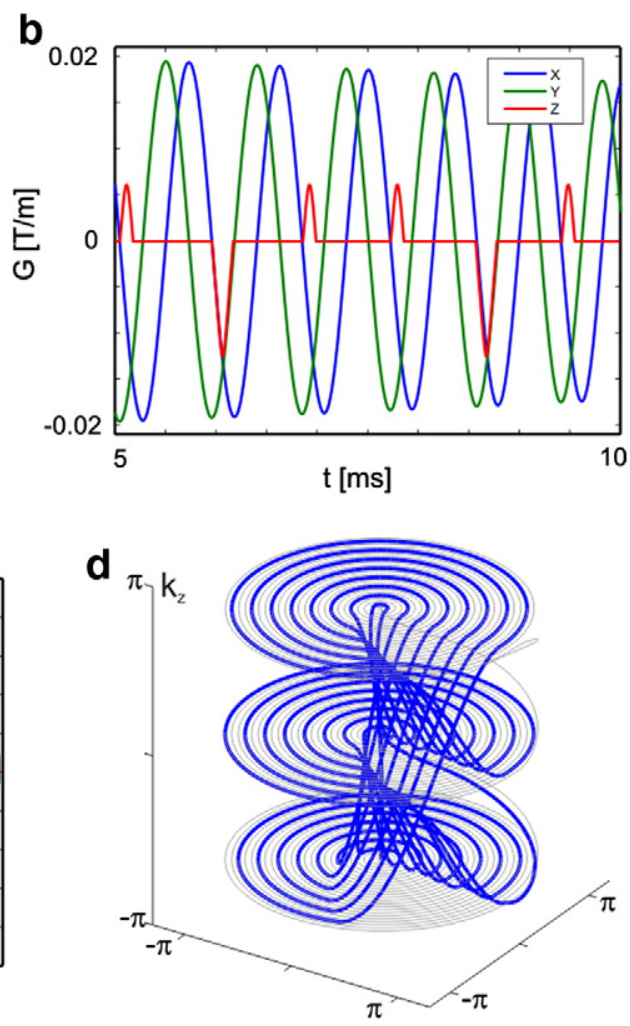

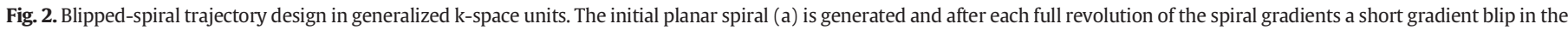

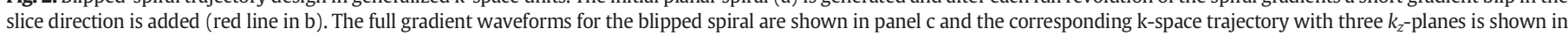
panel d. 


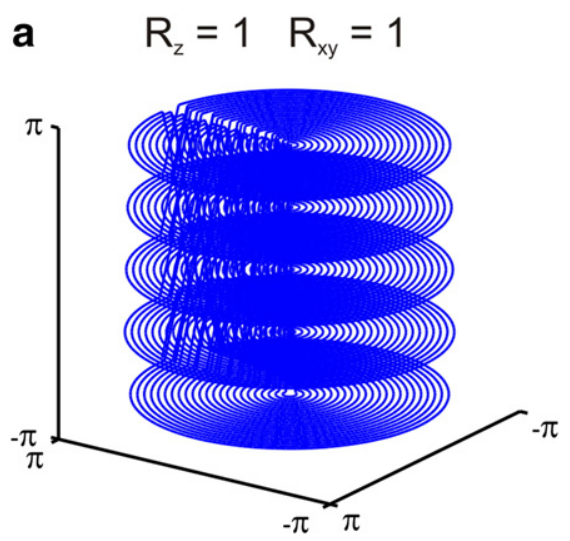

b $\quad \mathrm{R}_{\mathrm{z}}=5 \quad \mathrm{R}_{\mathrm{xy}}=1$
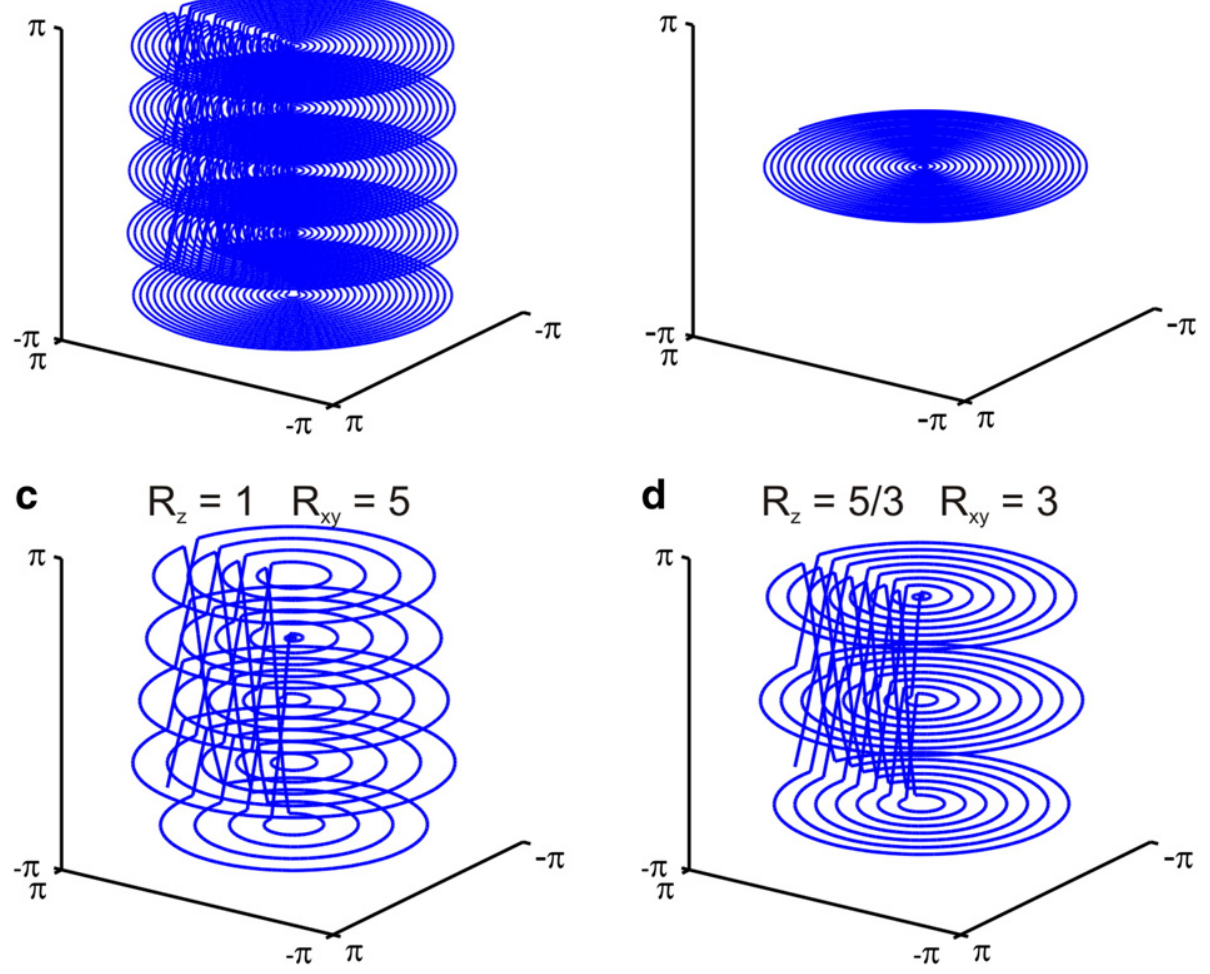

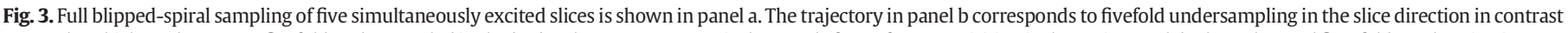

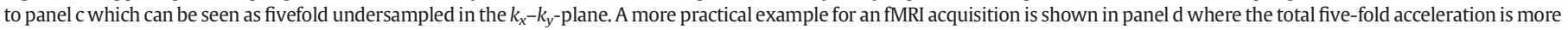
homogeneously distributed.

slice direction. This latter example is analogous to an SMS blipped-CAIPI EPI acquisition of five simultaneous slices with a FOV/3 shift between neighboring slices (also referred to as blip factor 3 ).

\section{Material and methods}

\section{Image acquisition}

All experiments were performed on a $3 \mathrm{~T}$ Tim Trio system (Siemens Healthcare, Erlangen, Germany) equipped with a 32-channel head coil array. Data were acquired in four healthy subjects after obtaining informed consent using an institutionally approved protocol. SMS excitation of $N$ slices was performed using a windowed multiband Sinc excitation pulse of $2.56 \mathrm{~ms}$ duration and with $N$ frequency bands. The z-gradient blip waveforms were designed by employing a linear programming algorithm for optimal gradient waveforms (Hargreaves et al., 2004) given a required k-space increment with gradient peak amplitude and slew rate constraints. For the 2D spirals optimal use of gradient slew rate was achieved by solving the differential equation describing the evolution of the slew rate vector (King et al., 1995). A spiral-in design was used because it offers optimal use of scan time and off-resonance behavior because the acquisition starts immediately after the excitation pulse and TE occurs at the end of the trajectory. To avoid peripheral nerve stimulation, the maximum slew rate for the design was set to $135 \mathrm{~T} / \mathrm{m} / \mathrm{s}$. The imaging parameters for a given blipped-spiral implementation are presented below as needed. All blipped-spiral trajectories were designed off-line and uploaded to the pulse sequence. The image reconstruction was performed offline. All gradient waveform designs, as well as the image reconstructions and fMRI data analyses described below, were performed using Matlab (The Mathworks, Natick, MA).

\section{Image reconstruction}

The method of conjugate gradients and a nuFFT gridding algorithm were used to reconstruct the blipped-spiral images (www.eecs.umich. edu/ fessler/irt/irt). Since no additional regularization was employed, it closely follows the generalized SENSE approach described in Pruessmann et al. (2001). Coil sensitivity and off-resonance maps were derived from a dual gradient echo (GRE) pre-scan covering the entire FOV spanned by the SMS acquisitions (FOV $=192 \times 192 \times 96 \mathrm{~mm}^{3}$, $\mathrm{TR}=168 \mathrm{~ms}, \mathrm{TE}_{1} / \mathrm{TE}_{2}=2.38 / 4.76 \mathrm{~ms}$, flip angle $\left.25^{\circ}\right)$. Off-resonance correction was implemented by a time-segmented formulation of the signal equation which approximates the continuous off-resonance induced phase evolution by piecewise constant phase maps and interpolates in between segments as proposed by Sutton et al. (2003). For this work Hanning window interpolation with ten time segments was used.

To examine the performance of the blipped-spiral trajectory three sets of images with the following parameters were acquired in a subject: (1) $3 \mathrm{~mm}$ isotropic resolution with FOV $=192 \times 192 \times$ $96 \mathrm{~mm}^{3}, N=8, \mathrm{TR}=168 \mathrm{~ms}, \mathrm{TE}=35 \mathrm{~ms}, R_{\mathrm{tot}}=5.1$ and $R_{z}=8 / 3$; (2) $2.5 \mathrm{~mm}$ isotropic resolution with FOV $=200 \times 200 \times 100 \mathrm{~mm}^{3}$, $N=8, \mathrm{TR}=215 \mathrm{~ms}$, TE $=35 \mathrm{~ms}, R_{\text {tot }}=6.4$ and $R_{z}=8 / 3$; and (3) $2 \mathrm{~mm}$ isotropic resolution with FOV $=192 \times 192 \times 96 \mathrm{~mm}^{3}, N=$ $8, \mathrm{TR}=294 \mathrm{~ms}, \mathrm{TE}=42 \mathrm{~ms}, R_{\mathrm{tot}}=7.5$ and $R_{z}=8 / 3$. Maps of the noise enhancement (g-factor maps) due to the accelerated imaging were then calculated using an approach inspired by the pseudo replica method in Robson et al. (2008). For a given accelerated k-space trajectory and 32-channel sensitivity maps, the raw data vector was replaced by a noise vector with uniform noise distribution (drawn from the interval $[-1 ; 1]$ assuming negligible noise correlation between coil elements) and reconstructed. This was repeated 50 times and the noise enhancement maps were given as the standard deviation of the noise for each pixel in the pseudo time series. In the same way noise 
enhancement maps were calculated for a non-accelerated blipped spiral trajectory which serves as a reference. The g-factor maps were then generated from the noise maps by pixel-wise division with the fully sampled noise map and accounting for the reduced number of sampled by dividing by the square root of the total acceleration factor. The g-factor maps then show the total noise enhancement relative to a fully sampled SMS 3D k-space and not only the contributions from multi-band acceleration.

\section{Comparison between blipped-spiral and undersampled stack-of-spirals}

The 3D k-space coverage of the blipped-spiral acquisition can also be accomplished by a single shot stacked-spiral trajectory (Assländer et al., 2013). In that work it was shown that changing the phase encoding direction along $z$, the direction of the strongest off-resonance gradients (e.g. above the sinuses), reduces off-resonance induced signal loss and blurring. In order to compare the off-resonance behavior of the stacked-spiral approach with the proposed blipped-spiral approach, trajectories of equal sampling time and approximately identical $\mathrm{k}$-space coverage where designed. Images were then acquired in a subject with the following parameters: FOV $=192 \times 192 \times 75 \mathrm{~mm}^{3}, N=5$, $\mathrm{TR}=200 \mathrm{~ms}, \mathrm{TE}=35 \mathrm{~ms}, R_{\mathrm{tot}}=4, R_{z}=1$. The trajectories are plotted in Figs. $4 \mathrm{c}$ and $\mathrm{g}$. In addition, $\mathrm{g}$-factor maps for the stacked-spiral and the blipped-spiral images were calculated as described above. No offresonance correction was employed in calculating the g-factor maps.

\section{Functional stimulation and statistical analysis}

Two visual activations paradigms were used to evaluate the utility of the blipped-spiral SMS sequence. The first was a flickering checkerboard task with a total duration of $60 \mathrm{~s}$. The task consisted of four blocks where each block consisted of $5 \mathrm{~s}$ of stimulation and $10 \mathrm{~s}$ of rest. During each stimulation period the subject was asked to perform bilateral finger tapping as well. In this manner the delay between the visual and motor a

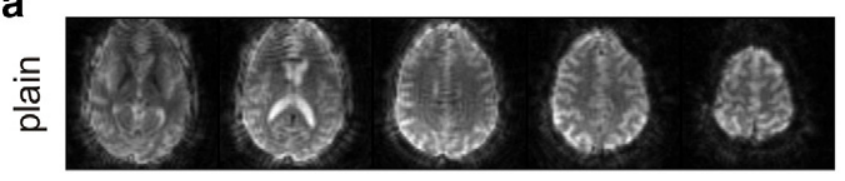

b

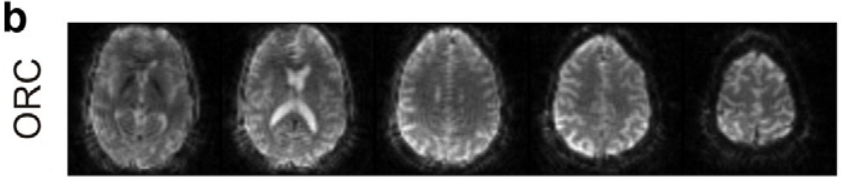

\section{d}

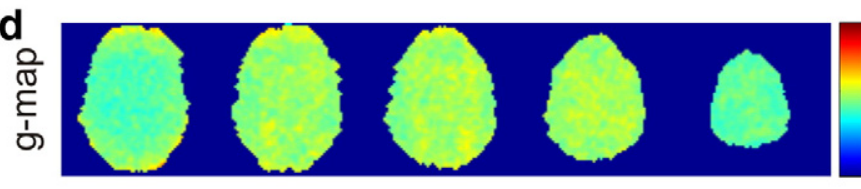

[\%]

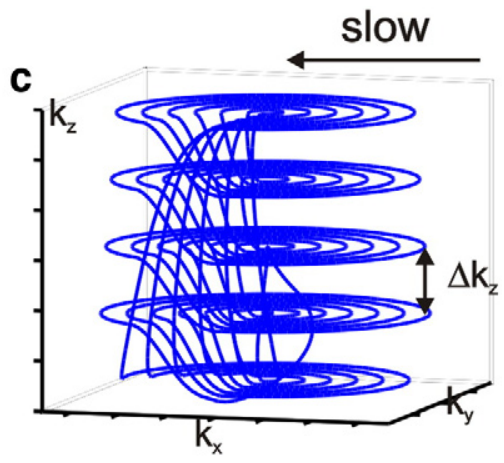

10 e

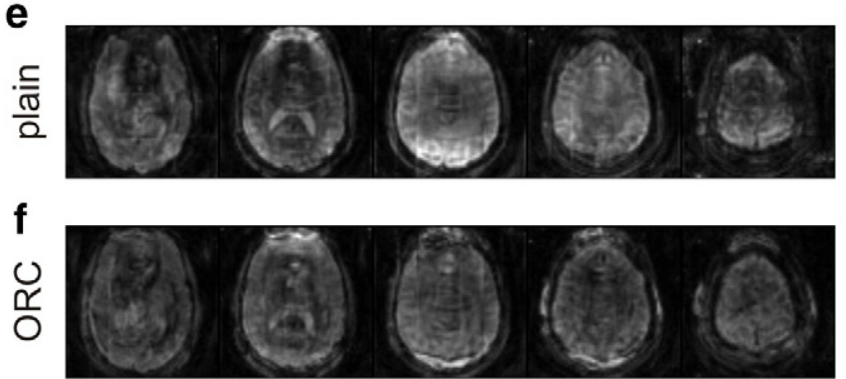

h

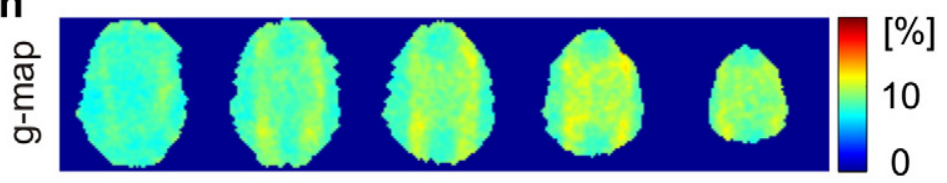

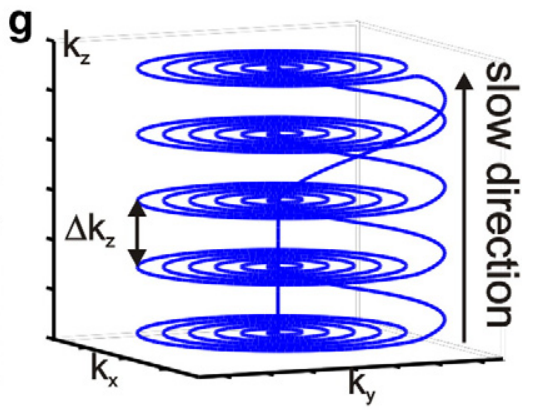

[\%]

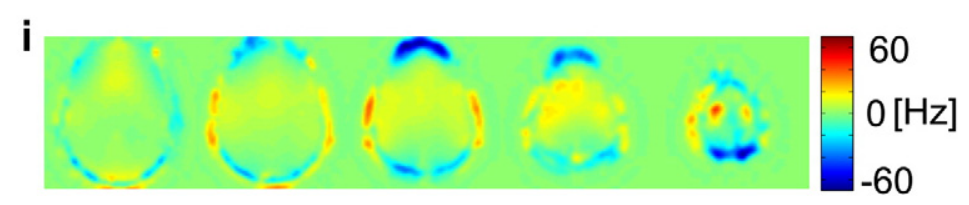

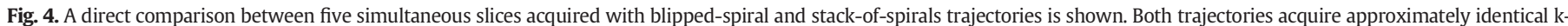

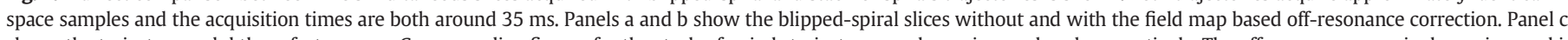

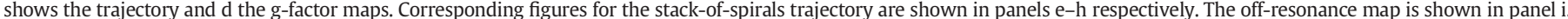

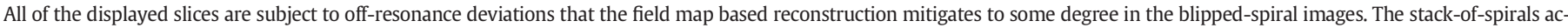

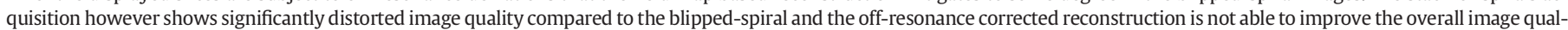
ity. The g-factor values are comparably low for both trajectories. 
responses could be measured. The functional images were acquired with $2 \mathrm{~mm}$ isotropic spatial resolution (FOV $=192 \times 192 \times 96 \mathrm{~mm}^{3}$, $N=8, \mathrm{TR}=294 \mathrm{~ms}, \mathrm{TE}=42 \mathrm{~ms}, R_{\mathrm{tot}}=7.5, R_{z}=8 / 3$ ).

The second paradigm was for dynamic eccentricity mapping of the visual cortex (Brefczynski and DeYoe, 1999). The stimulation consisted of expanding flickering rings (angular width $15^{\circ}$ ), with a periodicity of $18 \mathrm{~s}$ defined as the time from central to peripheral stimulation of the visual field (see Fig. 8a). One time series consisted of five stimulation cycles resulting in a total scan time of $90 \mathrm{~s}$. The functional time series was acquired using a FOV $=200 \times 200 \times 100 \mathrm{~mm}^{3}, N=8$, TR $=$ $215 \mathrm{~ms}, \mathrm{TE}=35 \mathrm{~ms}, R_{\mathrm{tot}}=6.7, R_{z}=8 / 3$ resulting in $2.5 \mathrm{~mm}$ isotropic spatial resolution.

Prior to the activation analysis the reconstructed time series was spatially smoothed with an isotropic Gaussian filter (kernel width $5 \mathrm{~mm}$ ) and slow drifts in the temporal domain were removed by subtracting linear and second order fits. No motion correction or realignment was performed. The ECG and respiration components of the time series were identified manually by performing a spectral analysis. The first and second order components were then removed by applying a notch filter in the spectral domain (filter width $\pm 0.1 \mathrm{~Hz}$ around each component's spectral peak). A general linear model (GLM) activation analysis was then performed with a canonical HRF, taken from SPM8 (www.fil.ion.ucl.ac.uk/spm/software/spm8) with default values, that was convolved with the stimulus onset function yielding the BOLD model function. The voxel-specific lag in the HRF delay was found by shifting the BOLD model in the GLM-analysis in steps of TR spanning $\pm 10 \times$ TR and calculating the corresponding T-map for every delay. For every voxel the optimal HRF delay was assumed to be given by the highest T-value along the delay domain. The first $18 \mathrm{~s}$ of each time series was discarded to allow a transition into a steady state, both for the MR signal and the BOLD response.

\section{Results}

\section{SMS imaging with blipped and stacked spiral trajectories}

Fig. 4 compares the five simultaneous slices acquired with the blipped-spiral trajectory (Figs. 4a-d) to the stacked-spiral approach (Figs. 4e-h). Figs. 4a and b show the result of blipped-spiral acquisitions, reconstructed without and with off-resonance correction (ORC). The blipped-spiral trajectory is shown in Fig. 4c and the corresponding g-factor maps are shown in Fig. 4d. The stacked-spiral trajectory images are shown without (Fig. 4e) and with (Fig. 4f) off-resonance correction as well. The stacked-spiral trajectory and corresponding g-factor maps are shown in Figs. $4 \mathrm{~g}$ and h. Data were acquired within a few seconds and the off-resonance maps displayed in Fig. 4i are hence valid for both scans.

The g-factor maps in Figs. $4 \mathrm{~d}$ and $\mathrm{h}$ demonstrate that both trajectories experience very low additional g-factor penalty (around 10\%) with slightly better performance by the stacked-spiral trajectory. One reason for the differences in g-factor penalty is the missing k-space samples during the transition period of the blipped spiral. The wedge shaped "holes" in k-space reduce the local sampling density and thus leading to increased g-factor noise. The total amount of off-resonance induced blurring should be approximately identical for both trajectories due to their similar lengths and sampling; however the stacked-spiral

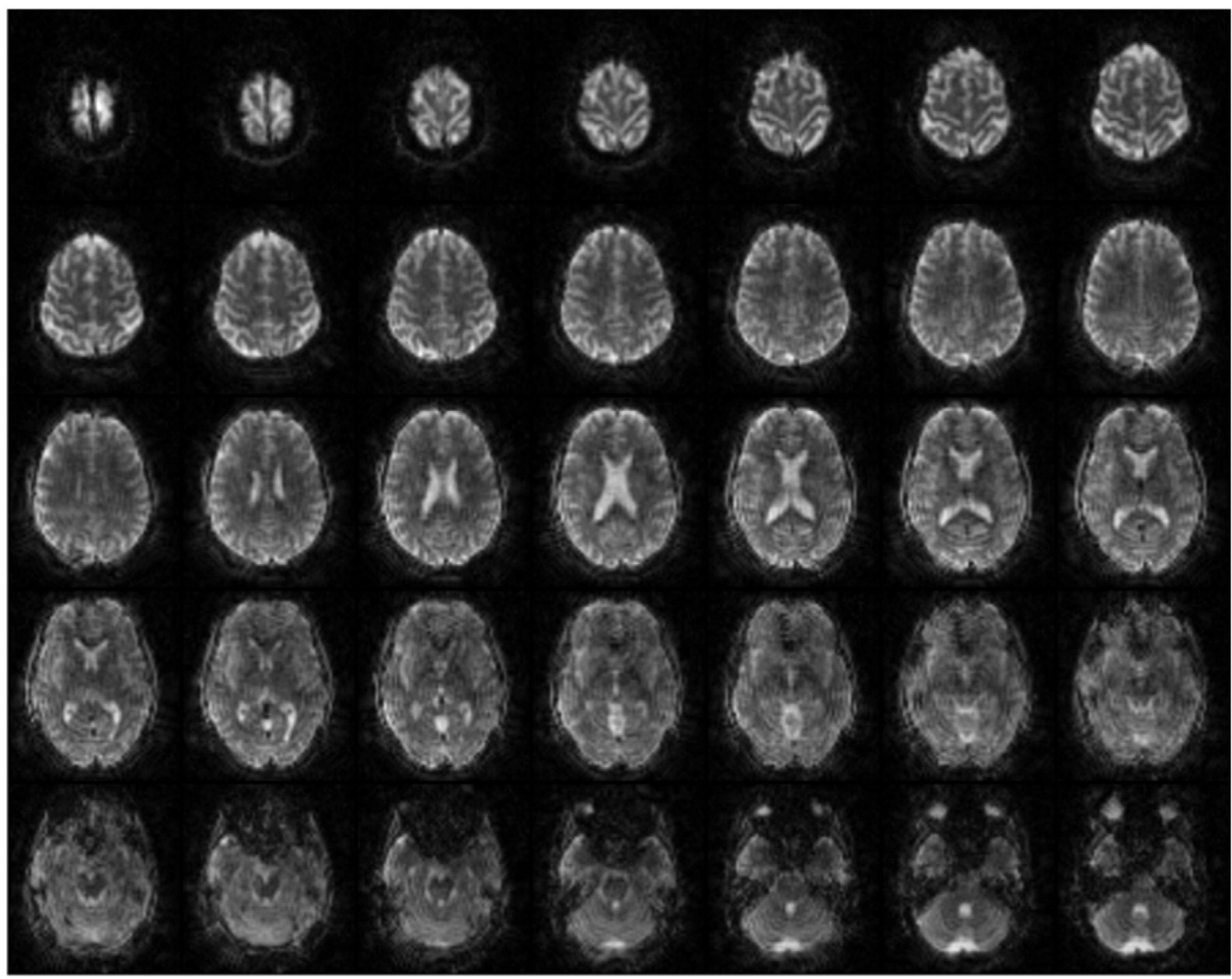

Fig. 5. This figure shows an example of 35 slices with $3 \mathrm{~mm}$ isotropic resolution acquired with five excitations in a total of $200 \mathrm{~ms}$ using an $N=7$ SMS blipped-spiral acquisition. 
images show significantly more off-resonance artifact compared to those obtained using the blipped-spiral. In both cases off-resonance leads to a more pronounced blurring of the point spread function (PSF) along the k-space dimension that is acquired slowly over the entire duration of the acquisition. For the stacked-spiral trajectory, blurring is predominantly along the slice-select direction and for the blipped-spiral it is along the in-plane directions. In-plane blurring of neighboring voxels is a local effect reducing the effective spatial resolution, whereas the through-plane blurring of simultaneously excited slices is a non-local effect since neighboring slices in the reconstructed volume are generally from different regions of the brain up to several centimeters apart.

\section{Accelerated imaging and g-factor noise}

Fig. 5 shows the reconstruction of 35 slices with $3 \mathrm{~mm}$ isotropic resolution is displayed as an example of a typical fMRI scan in which off-resonance correction was performed. The parameters for the blipped-spiral trajectory were: FOV $=192 \times 192 \times 105 \mathrm{~mm}^{3}, N=7$, $\mathrm{TR}=205 \mathrm{~ms}$, TE $=35 \mathrm{~ms}, R_{\mathrm{tot}}=7.5, R_{z}=8 / 3$. The total acquisition time for one volume was $200 \mathrm{~ms}$. Because of the relatively short readout time of only $35 \mathrm{~ms}$, off-resonance induced signal loss and blurring especially above the sinuses are mostly suppressed.

Fig. 6 shows a comparison of images from different blipped-spiral acquisitions and the corresponding g-factor maps for completeness. The examples represent acquisition parameters that we found to be feasible for high temporal resolution fMRI applications. The scans covered a $\mathrm{FOV}_{z}$ of approximately $100 \mathrm{~mm}$ in the slice direction, which in most fMRI applications provides sufficient coverage of the brain yet does not cover the brain completely. The first row of Fig. 6a shows eight fully encoded standard $(N=1)$ spiral reference slices with an isotropic resolution of $3 \mathrm{~mm}$. Fig. $6 \mathrm{~b}$ shows eight slices out of the 32 acquired slices with $3 \mathrm{~mm}$ isotropic spatial resolution using the blipped-spiral trajectory. The g-factor maps are displayed below the slices and show pronounced noise enhancement only for the two inner slices. For Fig. $6 \mathrm{c}$ the spatial resolution was increased to $2.5 \mathrm{~mm}$. The g-factor maps now show increased noise as expected for a higher total acceleration factor. For $2 \mathrm{~mm}$ spatial resolution and 48 slices (Fig. 6d) the acquisition time per excitation had to be increased to $42 \mathrm{~ms}$ to provide sufficient in-plane k-space sampling density due to the higher total acceleration factor. The g-factor penalty compared to the previous exampled is thus further increased. Despite the definition of in-plane

a

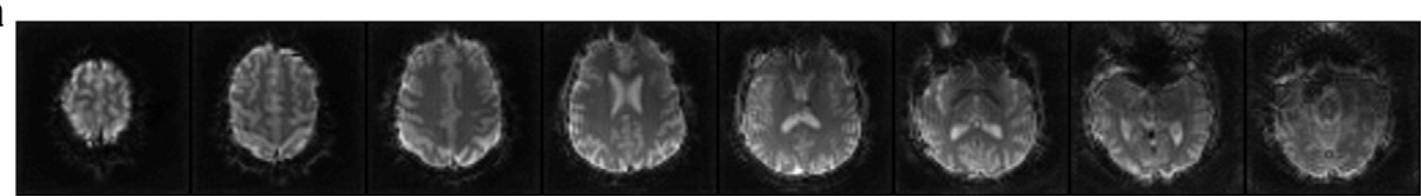

b
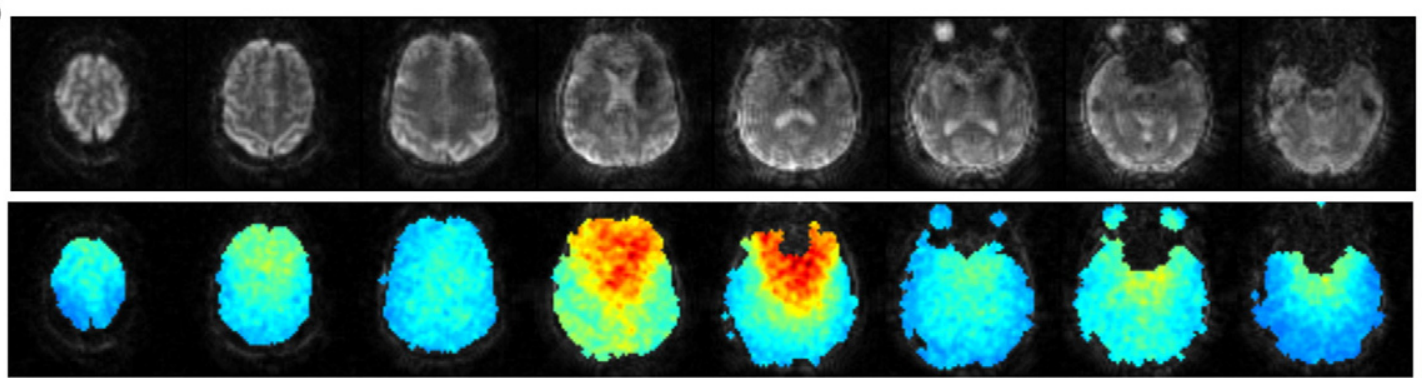

c

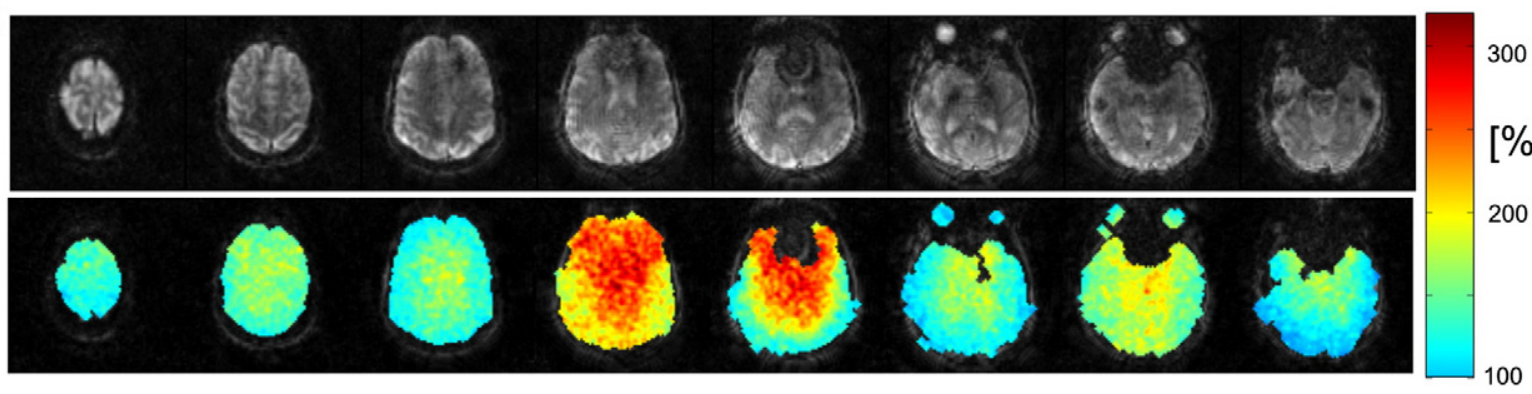

100

d
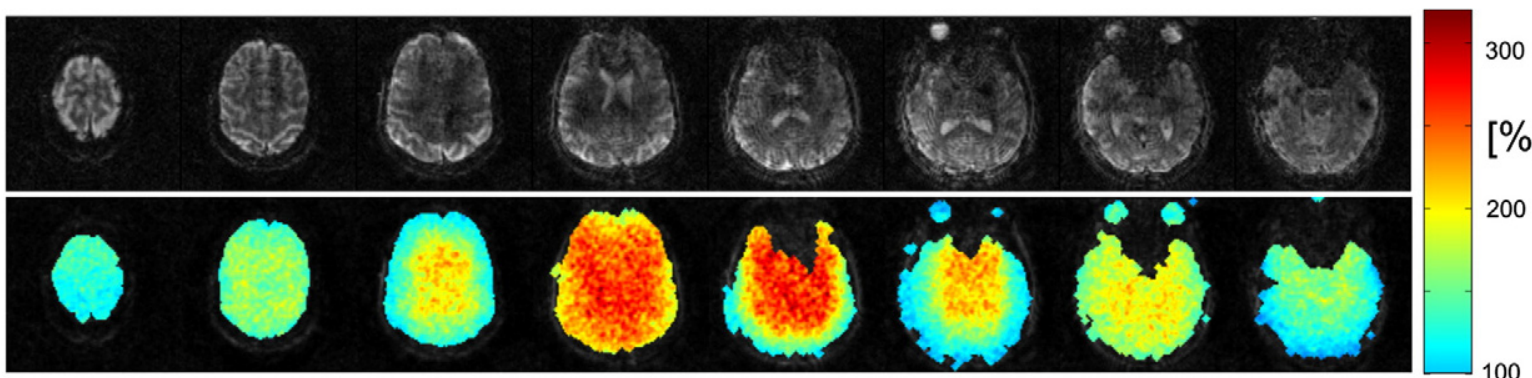

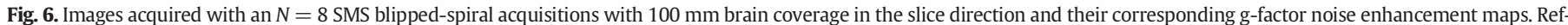

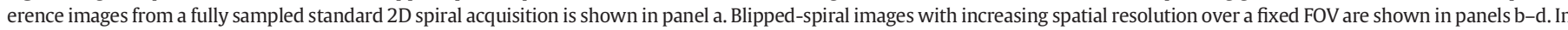

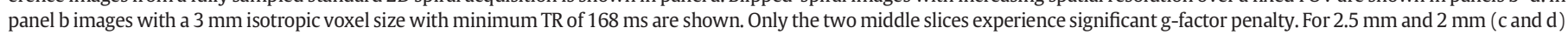
spatial resolutions the g-factor penalty increases as does the TR and the total acceleration factor (TR $=215 \mathrm{~ms}$ and $R_{\mathrm{tot}}=6.4$ for panel c; TR $=294 \mathrm{~ms}$ and $R_{\mathrm{tot}}=7.5$ for panel d). 
and multi-band acceleration factors, in general, a g-factor map contains contributions from both sampling directions depending on the undersampling pattern of the 3D k-space. Especially for higher slice acceleration factors $(>6)$ and additional in-plane undersampling, the 3D SMS k-space for both Cartesian and non-Cartesian sampling has the same properties as a regular k-space for 3D imaging.

The g-factor penalty generally is strongest in the center of the FOV as a result of the lower sensitivity variation of the receiver coil elements there. The higher g-factor values for the frontal regions of the brain can be explained with the opening of the helmet coil and the consequently lower coverage with individual coil elements.

\section{Visual/motor fMRI}

In Fig. 7 the results from the flashing checkerboard visual/motor stimulation task are shown. The functional maps are overlaid on the first volume of the time series and only the top and bottom 12 slices are displayed. The relative HRF onset for the motor cortex in Fig. 7a lags behind the visual cortex by $1-2 \mathrm{~s}$. The spatial variability of BOLD signal arrival times within the visual cortex was also previously shown in Zahneisen et al. (2011). The T-map corresponding to the GLM analysis with the optimal HRF delay is shown in Fig. 7b with an uncorrected Tthreshold of 4.5 (uncorrected p-value 0.000005 ). The average percentage BOLD signal time courses from the motor (red) and visual (blue) cortex in Fig. 7c also display the relative temporal shift of the motor response. The error bars display the standard error of all voxels that were averaged.

\section{Dynamic eccentricity mapping fMRI}

Fig. 8 shows the results from dynamic eccentricity mapping. The left row of Fig. 8a displays the temporal evolution of the flickering visual field stimulation pattern at various time points in steps of $4 \mathrm{~s}$. On the right, three voxel time courses together with the fitted BOLD response are plotted representing a short, medium, and long delay relative to the stimulation onset. The vertical dashed lines indicate the first maximum of the fitted BOLD model. Together with the intrinsic delay from the stimulation onset to the actual peak of the vascular response the phase of each voxel within the stimulation cycle can be calculated. Fig. 8b shows a map of the measured delay within the stimulation cycle $\Delta t_{\mathrm{HRF}}$ overlaying a structural T1 weighted scan that was coregistered to the blipped-spiral fMRI data. The parametric map only shows voxels with an uncorrected T-value above the chosen threshold of five (uncorrected p-value 0.00000041 ). The range of $\Delta t_{\mathrm{HRF}}$ corresponds to one stimulation cycle ( $18 \mathrm{~s}$ ). The characteristic cortex representation of the visual field (posterior for the focal part of the visual field and anterior for peripheral stimulation of the visual field) is clearly visible in this single-subject data.

\section{Discussion}

In summary we presented a novel blipped-spiral sequence for SMS imaging for fast fMRI. The method only requires the addition of an alternating blipped z-gradient, which is a simple modification to a standard 2D spiral imaging sequence. We did observe some loss in image quality
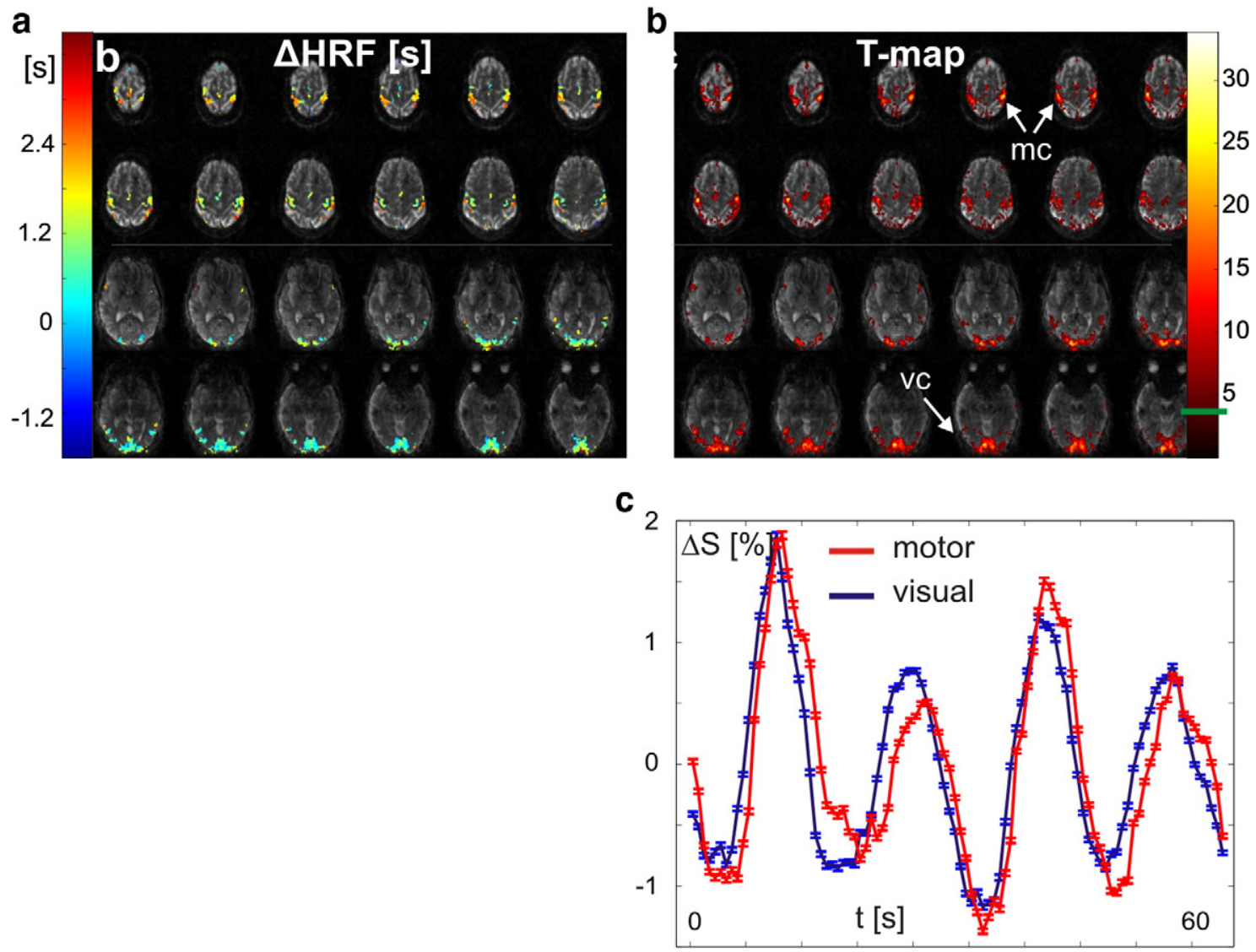

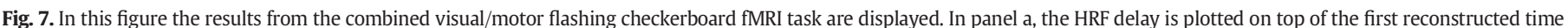

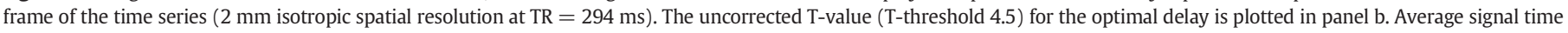

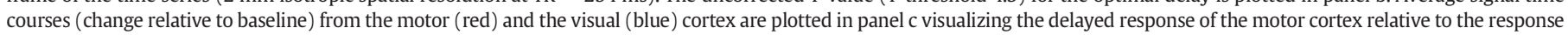
of the visual cortex. 
due to residual slice aliasing when compared to standard 2D spiral imaging as seen in Fig. 6. Compared to a 2D spiral readout for SMS excitation, however, we found that considerably reduced g-factors and more homogeneous g-factor distributions are achieved by distributing the 2D spiral trajectory over multiple planes using the gradient blips in the slice direction to effectively sample an SMS 3D k-space. This SMS 3D k-space picture allows for the straightforward extension of nonCartesian parallel imaging reconstruction methods to SMS imaging. Note that the SMS 3D k-space picture is completely general and applies to blipped-CAIPI EPI as well. The total spiral-in sampling duration and therefore the total in-plane resolution is restricted by the desired TE, which at $3 \mathrm{~T}$ is approximately $35 \mathrm{~ms}$ for maximum BOLD contrast. For the case of a 32-channel coil we found that $N=8$ slices could be acquired simultaneously if the spiral trajectory is distributed over $N_{k z}=3 \mathrm{k}$-space planes. If $N$ is further increased more k-space sampling density in slice direction is required. This can be achieved by distributing the spiral over more than three planes at the cost of in-plane sampling density of each plane since the total number of spiral rotations is a constant. Whole brain acquisition times for $3 \mathrm{~mm}$ isotopic resolution were $168 \mathrm{~ms}$ without dramatic g-factor penalty using these parameters. For $2 \mathrm{~mm}$ isotropic resolution the acquisition rate was less than $300 \mathrm{~ms}$, which is fast enough to at least capture the first harmonic of cardiac induced pulsatility. The imaging parameters for the examples shown were derived heuristically. A more optimal blipped-spiral trajectory certainly is a compromise between in-plane acceleration, number of slices, which affects the slice gap for a constant FOV in slice direction, and $\mathrm{k}$-space sampling density in the slice direction.

We have also shown that SMS acquisitions in fMRI have the advantage of improved temporal resolution of the BOLD HRF. This was demonstrated with the visual/motor flashing checkerboard task as well as with dynamic eccentricity mapping of the visual cortex. An eccentricity map with distinctive spatial features can be acquired from a single subject in less than 2 min using the blipped-spiral SMS acquisition. Because of the proof-of-concept nature of this experiment we did not calibrate for
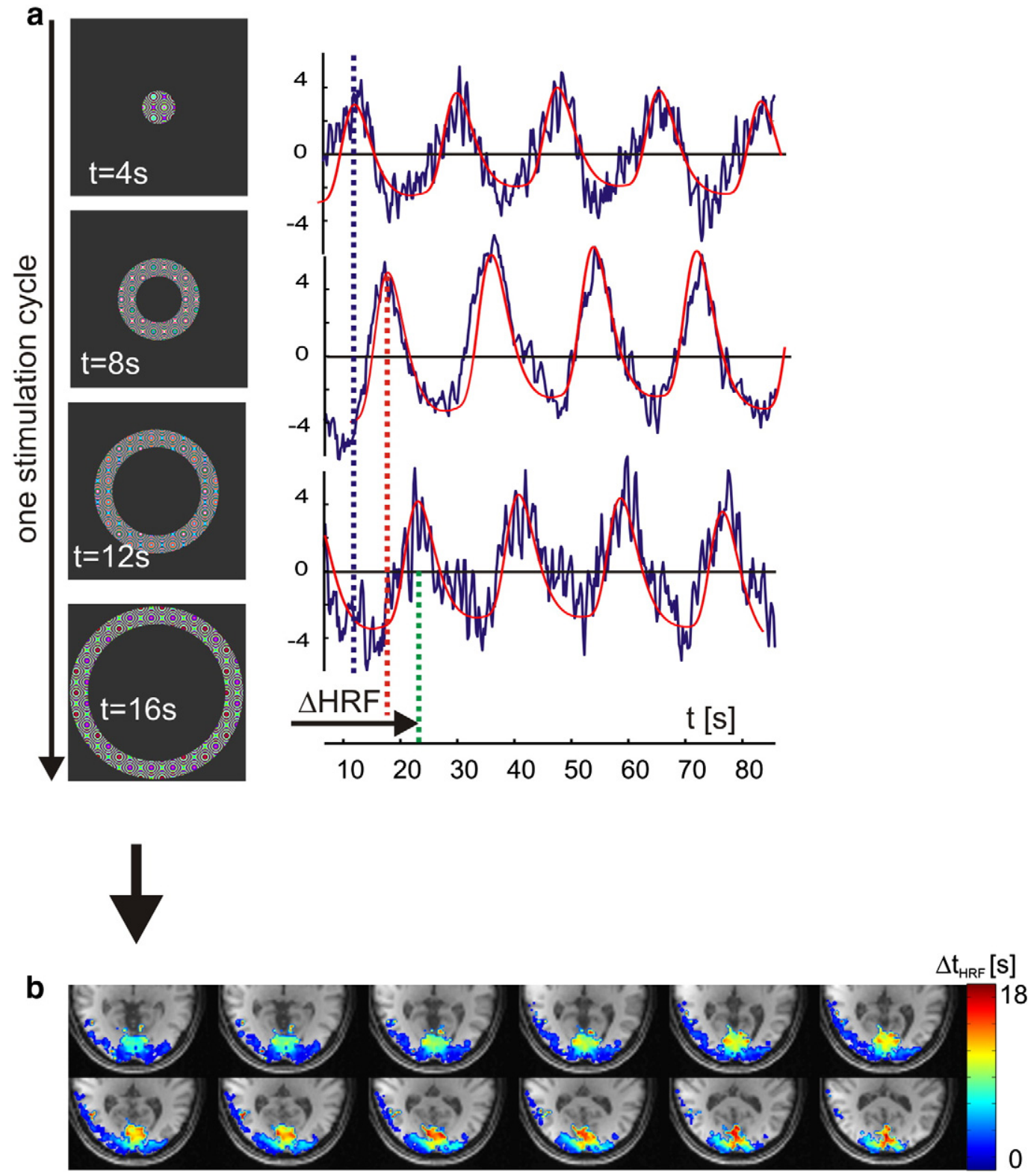

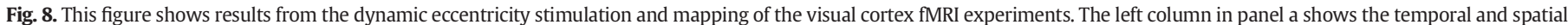

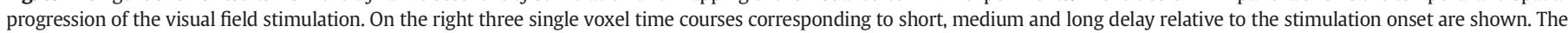

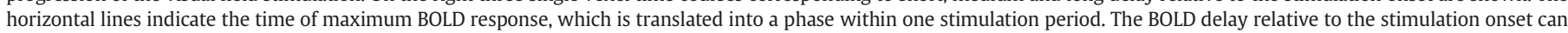
then directly be transformed into an eccentricity map of the visual cortex (b). 
locally varying vascular response delays by performing a second experiment with modified or inverted stimulation paradigm. The BOLD time courses in Fig. 8 show the direct recording of the HRF response, which allows the extraction of locally varying features like onset time or variations in shape. This also shows how the high temporal resolution simplifies the analysis of dynamics within the functional response. No jittering or multiple repetitions, which are based on the assumption of identical responses to identical stimuli over the course of an experiment, need to be employed to indirectly measure BOLD latencies. We expect that blipped-CAIPI EPI methods will show similar results.

The main advantage of the spiral approach compared to EPI is that the gradient utilization is more efficient, making optimal use of slewrate, and as a result spiral readouts are approximately $30 \%$ shorter when matched for coverage. Also the use of spiral-in makes use of all available time such that immediately after TE another group of simultaneous slices can be acquired. This produces improvement in the fMRI temporal resolution and increased sampling power. In terms of the maximum number of slices that can be acquired simultaneously using the blipped-spiral readout, we expect a very similar behavior as EPI based SMS approaches since both acquire the slice direction of k-space with Cartesian spacing. The main difference between spiral and EPI based acquisition is the PSF response to off-resonances. In EPI offresonances lead to a shift of the PSF along the slow phase encoding direction, which can be corrected in a post-processing step after the actual image reconstruction. For spiral like acquisition the PSF experiences inplane blurring which, if uncorrected, effectively reduces the spatial resolution. Additional acceleration in EPI can be also achieved using Partial Fourier techniques and Simultaneous Image Refocusing (SIR) (Feinberg et al., 2002). A direct comparison between spiral and EPI based acquisitions in terms of minimum volume TR however is beyond the scope of this work and needs to be investigated further.

Compared to other non-Cartesian 3D trajectories, like stacked-spiral approaches, the blipped-spiral trajectory avoids having the $z$ phase encoding along the slice direction as the slow direction. Although blipped-spiral has the in-plane dimension as the slow direction, advancing from the periphery to the center of the 3D k-space leads to in-plane blurring in contrast to through-slice blurring as in the case of a stack of $\mathrm{k}$-space planes that are acquired sequentially. The result is a significant improvement in image quality for the blipped-spiral trajectory compared to the stacked-spiral approach. The use of the spiral-in readout of only $35 \mathrm{~ms}$ duration keeps in-plane blurring at acceptable levels and contributes to the good overall image quality. We found no blipped-spiral g-factor improvement using the CAIPI style sampling compared to stacked-spirals, which actually showed slightly lower g-factors. This is likely due to the specific nature of spiral aliasing where the aliased signal is distributed over the complete FOV. Adding undersampled slices together therefore does not lead to a higher concentration of aliasing energy in the center of the image as in Cartesian sampling. Consequently, no improvement is expected by modifying the distribution of the spiral undersampling artifact in image space. The slightly higher g-factor for the blipped-spiral may be due to applying the $z$-gradients during the spiral readout creating unwanted undersampling. This g-factor penalty however is minimal compared to the gains in off-resonance robustness and blipped-spiral still produces images of superior quality compared to stacked-spirals. In practice it might be difficult to distinguish between pure g-factor effects on the image quality and signal dephasing due to local off-resonances when both effects have comparable magnitude. Furthermore recent work shows that off-resonance impacts g-factor measurements in phase constrained SMS reconstruction (Blaimer et al., 2013). Future investigation of the effects of off-resonance on SMS g-factor is needed. Additional improvements in image quality for high acceleration factors could be achieved by including regularization in the iterative image reconstruction (Block et al., 2007; Hugger et al., 2011).

The temporal resolution for the blipped-spiral for a $3 \mathrm{~mm}$ isotropic voxel size is only reduced by approximately a factor of two compared to the reported values for single shot 3D k-space acquisitions (100 ms for the latter case). However, the gain in image quality, the drastically reduced signal loss around the sinuses, and the effective spatial resolution being close to the nominal resolution outweigh the loss of temporal resolution compared to 3D single shot techniques. For the 32-channel receive coil array with its circular distribution of coil elements a homogenous (under)-sampling of the SMS 3D k-space seems promising. An advantage of SMS methods over 3D techniques is also that the separation between slices is a more efficient use of coil sensitivity variations, which allows greater acceleration along the slice direction compared to the in-plane directions. Because of the low volume TR both 3D and SMS methods have some signal loss due to the lower Ernst flip angle $\left(15-25^{\circ}\right)$ compared to an fMRI sequence with higher TR. This loss of in-plane SNR, however, is compensated by the ability to remove the fast physiological noise components by direct filtering and therefore increasing temporal SNR and by the unique ability to directly record the voxel specific HRF.

The blipped-spiral technique also has the flexibility of utilizing more sophisticated spiral sampling strategies. For example, further reduction in both g-factor penalty and acquisition times with a minimized tradeoff in spatial resolution can easily be achieved by replacing the spiral-in trajectory with a variable density spiral-in trajectory (Lee et al., 2003) where the periphery of k-space is sampled less densely than the center of k-space. An even more sophisticated approach would also allow for variable density sampling along the slice direction which can be achieved by reducing the k-space increment in the slice direction as the spiral moves to the center of k-space. Furthermore, the trajectory's $k_{z}$-component (slice direction), which in the current implementation follows a Cartesian sampling pattern, can be replaced by a continuously oscillating gradient in the slice direction as done by Chu and Noll (2013). The effectiveness of these approaches, however, has to be further investigated.

\section{Acknowledgments}

This work was supported by funding from the NIH (1R01 DA021146 (BRP), U54 56883 (SNRP) and R01DA019912, R01EB011517, and K02DA020569). BPA was partly funded by DFG grant Po1576/1-1.

\section{References}

Assländer, J., Zahneisen, B., Hugger, T., Reisert, M., Lee, H.-L., LeVan, P., Hennig, J., 2013. Single shot whole brain imaging using spherical stack of spirals trajectories. Neuroimage 73, 59-70 (Jun).

Blaimer, M., Choli, M., Jakob, P.M., Griswold, M.A., Breuer, F.A., 2013. Multiband phaseconstrained parallel mri. Magn. Reson. Med. 69 (4), 974-980 (Apr).

Block, K.T., Uecker, M., Frahm, J., 2007. Undersampled radial MRI with multiple coils. Iterative image reconstruction using a total variation constraint. Magn. Reson. Med. 57 (6), 1086-1098 (Jun).

Brefczynski, J.A., DeYoe, E.A., 1999. A physiological correlate of the 'spotlight' of visual attention. Nat. Neurosci. 2 (4), 370-374 (Apr).

Breuer, F.A., Blaimer, M., Mueller, M.F., Seiberlich, N., Heidemann, R.M., Griswold, M.A., Jakob, P.M., 2006. Controlled aliasing in volumetric parallel imaging (2D CAIPIRINHA). Magn. Reson. Med. 55 (3), 549-556 (Mar).

Chen, L., An, V., Xu, J., Ugurbil, K., Yacoub, E., Feinberg, D.A., 2013. Highly accelerated EPI evaluated for fMRI. Proc. Int. Soc. Magn. Reson. Med. 21.

Chu, A., Noll, D.C., 2013. Simultaneous multislice spiral imaging using z-gradient modulation and parallel receive coils. Proc. Int. Soc. Magn. Reson. Med. 21, 3317.

Dilharreguy, B., Jones, R.A., Moonen, C.T.W., 2003. Influence of fMRI data sampling on the temporal characterization of the hemodynamic response. Neuroimage 19 (4), 1820-1828 (Aug)

Feinberg, D.A., Setsompop, K., 2013. Ultra-fast MRI of the human brain with simultaneous multi-slice imaging. J. Magn. Reson. 229, 90-100 (Apr).

Feinberg, D.A., Reese, T.G., Wedeen, V.J., 2002. Simultaneous echo refocusing in EPI. Magn. Reson. Med. 48 (1), 1-5 (Jul).

Feinberg, D.A., Moeller, S., Smith, S.M., Auerbach, E., Ramanna, S., Gunther, M., Glasser, M.F., Miller, K.L., Ugurbil, K., Yacoub, E., 2010. Multiplexed echo planar imaging for sub-second whole brain fMRI and fast diffusion imaging. PLoS One 5 (12), e15710.

Glover, G.H., 2012. Spiral imaging in fMRI. Neuroimage 62 (2), 706-712 (Aug).

Griswold, M.A., Jakob, P.M., Heidemann, R.M., Nittka, M., Jellus, V., Wang, J., Kiefer, B., Haase, A., 2002. Generalized autocalibrating partially parallel acquisitions (GRAPPA). Magn. Reson. Med. 47 (6), 1202-1210 (Jun).

Hargreaves, B.A., Nishimura, D.G., Conolly, S.M., 2004. Time-optimal multidimensional gradient waveform design for rapid imaging. Magn. Reson. Med. 51 (1), 81-92 (Jan). 
Hugger, T., Zahneisen, B., Levan, P., Lee, K.J., Lee, H.-L., Zaitsev, M., Hennig, J., 2011. Fast undersampled functional magnetic resonance imaging using nonlinear regularized parallel image reconstruction. PLoS One 6 (12), e28822.

Irarrazabal, P., Nishimura, D.G., 1995. Fast three dimensional magnetic resonance imaging. Magn. Reson. Med. 33 (5), 656-662 (May).

King, K.F., Foo, T.K., Crawford, C.R., 1995. Optimized gradient waveforms for spiral scanning. Magn. Reson. Med. 34 (2), 156-160 (Aug).

Larkman, D.J., Hajnal, J.V., Herlihy, A.H., Coutts, G.A., Young, I.R., Ehnholm, G., 2001. Use of multicoil arrays for separation of signal from multiple slices simultaneously excited. J. Magn. Reson. Imaging 13 (2), 313-317 (Feb).

Lee, J.H., Hargreaves, B.A., Hu, B.S., Nishimura, D.G., 2003. Fast 3D imaging using variabledensity spiral trajectories with applications to limb perfusion. Magn. Reson. Med. 50 (6), 1276-1285 (Dec).

Moeller, S., Yacoub, E., Olman, C.A., Auerbach, E., Strupp, J., Harel, N., Ugurbil, K., 2010. Multiband multislice GE-EPI at 7 Tesla, with 16-fold acceleration using partial parallel imaging with application to high spatial and temporal whole-brain fMRI. Magn. Reson. Med. 63 (5), 1144-1153 (May).

Nunes, R., Hajnal, J.V., Golay, X., Larkman, D.J., 2006. Simultaneous slice excitation and reconstruction for single shot EPI. Proceedings of the 14th annual meeting of ISMRM, Seattle, p. 293.

Posse, S., Ackley, E., Mutihac, R., Rick, J., Shane, M., Murray-Krezan, C., Zaitsev, M., Speck, O., 2012. Enhancement of temporal resolution and bold sensitivity in real-time fMRI using multi-slab echo-volumar imaging. Neuroimage 61 (1), 115-130 (May).

Pruessmann, K.P., Weiger, M., Scheidegger, M.B., Boesiger, P., 1999. SENSE: sensitivity encoding for fast MRI. Magn. Reson. Med. 42 (5), 952-962 (Nov).

Pruessmann, K.P., Weiger, M., Börnert, P., Boesiger, P., 2001. Advances in sensitivity encoding with arbitrary k-space trajectories. Magn. Reson. Med. 46 (4), 638-651 (Oct).

Riemenschneider, B., Assländer, J., Hennig, J., 2013. An approach to 3-dimensional multiband acquisition. Proc. Int. Soc. Magn. Reson. Med. 21, 411.
Robson, P.M., Grant, A.K., Madhuranthakam, A.J., Lattanzi, R., Sodickson, D.K., McKenzie, C.A., 2008. Comprehensive quantification of signal-to-noise ratio and g-factor for image-based and k-space-based parallel imaging reconstructions. Magn. Reson. Med. 60 (4), 895-907 (Oct).

Setsompop, K., Gagoski, B.A., Polimeni, J.R., Witzel, T., Wedeen, V.J., Wald, L.L., 2012 Blipped-controlled aliasing in parallel imaging for simultaneous multislice echo planar imaging with reduced g-factor penalty. Magn. Reson. Med. 67 (5), 1210-1224 (May).

Sodickson, D.K., Manning, W.J., 1997. Simultaneous acquisition of spatial harmonics (SMASH): fast imaging with radiofrequency coil arrays. Magn. Reson. Med. 38 (4) 591-603 (Oct).

Sutton, B.P., Noll, D.C., Fessler, J.A., 2003. Fast, iterative image reconstruction for MRI in the presence of field inhomogeneities. IEEE Trans. Med. Imaging 22 (2), 178-188 (Feb).

Wiggins, G.C., Triantafyllou, C., Potthast, A., Reykowski, A., Nittka, M., Wald, L.L., 2006. 32channel 3 Tesla receive-only phased-array head coil with soccer-ball element geometry. Magn. Reson. Med. 56 (1), 216-223 (Jul).

Witzel, T., 2008. Single-shot echo volumar imaging using highly parallel detection. Proceedings of the ISMRM 2008, Toronto.

Zahneisen, B., Grotz, T., Lee, K.J., Ohlendorf, S., Reisert, M., Zaitsev, M., Hennig, J., 2011 Three-dimensional mr-encephalography: fast volumetric brain imaging using rosette trajectories. Magn. Reson. Med. 65 (5), 1260-1268 (May).

Zahneisen, B., Hugger, T., Lee, K.J., LeVan, P., Reisert, M., Lee, H.-L., Assländer, J., Zaitsev, M., Hennig, J., 2012. Single shot concentric shells trajectories for ultra fast fMRI. Magn. Reson. Med. 68 (2), 484-494 (Aug).

Zahneisen, B., Poser, B.A., Ernst, T., Stenger, V.A., 2013. Three-dimensional Fourier encoding of simultaneously excited slices: Generalized acquisition and reconstruction framework. Magn. Reson. Med. http://dx.doi.org/10.1002/mrm.24875 (in press).

Zhu, K., Kerr, A., Pauly, J.M., 2013. Simultaneous multi-slice flyback echo planar imaging with auto-calibration. Proc. Int. Soc. Magn. Reson. Med. 21, 125. 